\title{
Heterogeneity spacers in 16S rDNA primers improve analysis of mouse gut microbiomes via greater nucleotide diversity
}

Elizabeth A Jensen ${ }^{1,2,3}$, Darlene E Berryman"1,3,4 , Erin R Murphy ${ }^{1,4,5,6}$, Ronan K Carroll1,7, Joshua Busken ${ }^{3,8}$, Edward O List ${ }^{1,3,9}$ \& William H Broach ${ }^{\star, 10}$

\section{ABSTRACT}

Illumina-based amplicon sequencing suffers from the deleterious effects of highly homogenous nucleotide composition, limiting the number of highquality reads generated per run. We attempted to alleviate this limitation by comparing the results obtained from 16S ribosomal DNA (16S rDNA) sequencing of mouse gut microbiomes using Illumina V3-V4 primers (Run 1) and custom primers that incorporate a heterogeneity spacer (0-7 nucleotides) upstream of the $16 \mathrm{~S}$ priming region (Run 2). Overall, Run 2 had higher quality sequences, a more diverse microbial profile, and higher precision within, and variation between, experimental groups than Run 1. Our primer design offers a simple way to increase the quality of 16S rDNA sequencing and increases the number of useable reads generated per Illumina run.

\section{METHOD SUMMARY}

The standard Illumina primers for 16S rDNA sequencing of the $\mathrm{V} 3-\mathrm{V} 4$ region were modified by adding heterogeneity spacer regions ( $0-7$ nucleotides) upstream of the 16S rDNA specificity binding region, which improved the effectiveness of the sequencing reaction as measured by sequence quality, increased sensitivity and increased observed variation in microbial abundance and diversity of the samples.

\section{KEYWORDS}

16S rDNA sequencing $\cdot$ microbiome $\cdot$ optimization - primer design $\cdot \mathrm{V} 3-\mathrm{V} 4$ region

'Department of Translational Biomedical Sciences, Graduate College, Ohio University, Athens, $\mathrm{OH}$, USA; ${ }^{2}$ Ohio University Heritage College of Osteopathic Medicine, Athens, $\mathrm{OH} 45701$, USA; ${ }^{3}$ The Diabetes Institute, Konneker Research Center 108, Ohio University, Athens, OH 45701, USA; ${ }^{4}$ Department of Biomedical Sciences, Heritage College of Osteopathic Medicine, Ohio University, Athens, OH 45701, USA; ${ }^{5}$ Molecular \& Cellular Biology Program, Ohio University, Athens, $\mathrm{OH} 45701$ USA; ${ }^{6}$ Infectious \& Tropical Disease Institute, Irvine 228, Ohio University, Athens, OH 45701, USA; 7 Department of Biological Sciences, College of Arts \& Sciences, Ohio University, Athens, $\mathrm{OH} 45701$, USA; ${ }^{8}$ College of Health Sciences \& Professions, Ohio University, Athens, OH 45701, USA; ${ }^{9}$ Edison Biotechnology Institute, Athens, $\mathrm{OH} 45701$, USA; ${ }^{10}$ Ohio University Genomics Facility, Ohio University, Athens, $\mathrm{OH} 45701$, USA; *Author for correspondence: broach@ohio.edu

BioTechniques 67: 55-62 (August 2019) 10.2144/btn-20190025
Research on the gut microbiome has increased exponentially over the last decade, elucidating links between the microbiota and human health [1,2]. Accuracy in profiling microbial communities, especially unculturable bacteria in the gut, is crucial and depends upon standardization and improved quality of analyses [3]. This fact is particularly true for 16S rDNA sequencing with Illumina MiSeq, which has helped facilitate research on the microbiome due to its capacity for high-throughput sequencing and long reads, with low analytic cost [4]. The 16S rDNA gene consists of nine hypervariable regions flanked by highly conserved regions, making it an attractive target for amplicon sequencing of microbial communities [5]. Moreover, the sequences of the hypervariable regions allow for identification of unique bacteria.

16S rDNA sequencing relies upon the specificity and accuracy of the primers designed to amplify specific hypervariable regions. Several studies have compared the microbial composition and diversity across different 16S rDNA primers, demonstrating that the level of taxonomic resolution detected varies depending on the hypervariable region targeted [6-9]. The V3-V4 16S rDNA primers have been commonly used across the microbiome field for soil, various animal models and humans $[6,7,10,11]$. As compared with sequencing other hypervariable regions, the V3-V4 hypervariable region provides a broad taxonomic range of bacteria, thus capturing more microbial diversity with decreased taxonomic bias $[6,7,12]$. For these reasons, many $16 \mathrm{~S}$ rDNA-based microbiome analyses are carried out using primers designed and optimized by Illumina to amplify the $\mathrm{V} 3-\mathrm{V} 4$ region of mixed microbial populations.

Despite the advantages in $16 \mathrm{~S}$ rDNA sequencing with Illumina V3V4 region primers, inherent limitations exist in an amplicon sequencing approach, including low nucleotide diversity of the amplicon and inherent error rate. Given the importance for taxonomic identification of each nucleotide within the $16 \mathrm{~S}$ rDNA amplicon, miscalling of one base increases the probability of misidentifying the bacterium [10]. To address these shortcomings in the standard Illumina V3-V4 primers, different techniques have been employed to increase the quality of the data, including quality trimming, read overlapping, targeting multiple hypervariable regions and dualindexing of the samples to reduce error rates $[4,10,13-16]$. The aforementioned techniques, however, do not address the inherent issue of low nucleotide diversity. The 16S rDNA primers recognize highly conserved regions that flank hypervariable regions, subsequently yielding amplicons with highly homogenous sequences. Moreover, the clustering technique and fluorescent identification inherent to Illumina technology can make it difficult to discern sequences with long repeats, inverted repeats or GGC $[10,13]$. Bases A and $C$ are more prone to substitution errors [13]. The first few amplification cycles are crucial for identification of the sequencing clusters on the flow cell, with misidentification resulting in loss of sequencing reads, low sequence quality and inaccurate base identification, all errors that alter the observed microbial profile. 
- Increased sequence variability, such as pairing the pool of samples with a heterogeneous control library like PhiX, counteracts these well-known problems, allowing for more accuracy in cluster identification and thereby, accuracy of microbial profiling. Unfortunately, the use of the PhiX control sacrifices sequencing space on the flow cell.

Another approach to counteract the issue of low nucleotide diversity is the addition of heterogeneity spacers, a nucleotide region, independent of the binding specificity or adapter regions required for Illumina sequencing chemistry, which serves to increase diversity within each sequencing cycle by offsetting the targeted nucleotides. Several 16S rDNA sequencing studies with heterogeneity spacers have shown increased sequence variability, better sequence quality and more reproducible datasets $[14,15]$. These studies, however, used a combination of multiple indexing and heterogeneity spacers to counteract both error rates and low nucleotide diversity. Our primer design focuses on the use of heterogeneity spacers to address high error rates and low nucleotide diversity of standard Illumina V3-V4 16S rDNA primers. This primer design uses shorter heterogeneity spacers and fewer primer sets (10 primer sets) than previous studies (22 primer sets). Our primer design adds nucleotide variation and ameliorates quality issues, thus allowing more sequences to pass the initial quality control (QC) stage of analysis. Using conserved mouse microbial samples, we compared sequence quality, primer effectiveness, microbial abundance and diversity between our novel primers and our controls, the standard Illumina V3-V4 primers.

\section{MATERIALS \& METHODS}

\section{Mouse fecal samples}

Animal experiments were conducted in accordance with the animal care and use protocol approved by the Institutional Animal Care and Use Committee of Ohio University and fully complied with federal, state and local policies. Female C57BL/6J mice at 6 months of age were used. Housing conditions, handler and time of fecal collection were controlled across groups. Mice were kept on a 14-h light/10-h dark cycle and had ad libitum access to water and normal chow (Prolab RMH 3000). Only six mouse fecal samples (a subset of the original, larger study with 192 fecal samples collected and loaded in Run 1 and of 100 fecal samples loaded in Run 2) were analyzed to compare the standard Illumina primers (Run 1) to our primer sets (Run 2). This sample size was chosen due to the small sample number in Run 1 that successfully passed initial QC analysis.

\section{Fecall collections}

Fecal pellets were collected at $9 \mathrm{am}$. Mice were allowed to defecate or, after $10 \mathrm{~min}$, encouraged to defecate through gentle massage of the hind-back region. The first pellet produced by each mouse was used for DNA isolation. Pellets were collected with sterilized forceps, weighed and immediately frozen on dry ice. Equipment and bench surfaces were sterilized with diluted bleach solution (1:10) between each collection. Frozen fecal pellets were stored in $-80^{\circ} \mathrm{C}$ freezers until further use.

\section{DNA isolation}

Total DNA was isolated using the MoBio PowerFecal DNA Isolation Kit according to the manufacturer's instructions (currently Qiagen's QIAamp PowerFecal DNA Isolation kit, Cat\#12830-50). DNA quality and quantity were measured using NanoDrop Spectrophotometer 2000c (Thermo Scientific).

\section{S rDNA amplicon library preparation}

The same extracted DNA was used for Runs 1 and 2. Samples for Run 1 were prepared following the protocol provided by Illumina (Part\# 15044223, Rev B). Samples for Run 2 were prepared using our novel primers, adapted from the original Illumina primer design with unique oligonucleotides that incorporate the Illumina adaptor overhang, a spacer region and V3-V4 specific primer (Figure 1 and Table 1). All 100 samples (5 ng of total DNA) were amplified with 10 oligonucleotide sets ( $0.2 \mu \mathrm{M}$ final concentration) in separate reactions (960 total reactions) using the Kapa Hifi HotStart PCR Kit (Kapa Biosystems, KK2502); 96 reaction products were assayed using an Agilent 2100 Bioanalyzer DNA 1000 chip (Agilent, 5067-1504). After confirmation of correctly sized products ( $550 \mathrm{bp})$, equimolar aliquots of each reaction were pooled by sample. Amplicons were then purified using Agencourt AMPure XP beads (BeckmanCoulter, A63881).
For both runs, purified libraries were barcoded using the Nextera XT Index Kit v2 (Illumina, FC-131-2001) according to the manufacturer's protocol. Final libraries were assayed for fragment size using the Agilent 2100 Bioanalyzer DNA 1000 chip (Agilent, 5067-1504) and quantified using the Qubit dsDNA BR Assay (Invitrogen, Q32853) on a Qubit 3.0 Fluorometer.

\section{S rDNA amplicon sequencing with} Illumina

Individually barcoded samples were pooled equimolarly and spiked with $10 \%$ PhiX to generate the multiplexed library to be sequenced on an Illumina MiSeq using the MiSeq Reagent Kit v3 (600-cycle; Illumina, MS-102-3003) $2 \times 300$ bp paired-end sequencing kit with a loading concentration of $10 \mathrm{pM}$

\section{Data analysis}

The resultant FASTQ files were analyzed using QIIME 2.0 and CLC Genomics Workbench with the Microbial Genomics Module, Version 11.0 and the pre-defined workflows for Data QC and Taxonomic Profiling (QIAGEN, CLC Bio, Aarthas, Denmark) [17]. Sequences were classified using operational taxonomical unit (OTU) clustering or picking with the $97 \%$ SILVA reference library [18].

With QIIME 2.0, samples in Run 1 were unexpectedly classified as chimeras and failed analysis; thus, microbial abundance and diversity were quantified for Run 1 and 2 with CLC Genomics Workbench. Microbial abundance from the phyla to genus level was analyzed between groups in each run ( $n=3$ per group) and compared between Run 1 and 2 ( $n=6$ per run). Diversity was assessed through alpha and beta diversity metrics, analyzing between experimental groups and between runs. Statistical significance was determined with $p<0.05$ using PERMANOVA (Bonferroni) and FDR $p$-value on CLC Genomics Workbench version 11.0 (QIAGEN, CLC Bio).

\section{Verification of primer effectiveness}

Primer effectiveness was evaluated from the output on sequence quality and OTU abundance output from the sequences. Primer coverage was assessed by in silico analysis using Ribosomal Database Project (RDP), which uses the paired 16S rDNA 
primers to assess a ratio of the total number of bacteria covered in each phylum and genus over the total known bacteria. A percentage was then created to approximate primer coverage for the primers used in both runs. Quality of each run was quantified through the average PHRED quality (Q) score of each nucleotide base and the percentage of successful microbial analysis. Primer effectiveness was also tested on the total number of reads, OTU reads before microbial analysis and percentage of sequence retention from total number of reads to OTU reads.

\section{RESULTS \& DISCUSSION}

We developed novel primers with added heterogeneity spacers to counteract wellknown limitations of low nucleotide diversity and inherent error rate associated with the use of standard Illumina V3-V4 primers. The addition of heterogeneity spacers introduced sequence heterogeneity in the first seven nucleotides, allowing for better identification of clusters in the first few cycles. Additionally, the spacers ameliorate quality issues that arise from each cluster fluorescing in the same channel.

Analysis was attempted with both OTU picking and denoising with QIIME 2.0 and OTU clustering with CLC Genomics Workbench. Run 2 was successfully analyzed with both methods. Samples in Run 1 failed initial QC and were classified as chimeras with QIIME 2.0 but could be processed in CLC Genomics Workbench. Consequently, a comparison between Run 1 (as controls) and Run 2 was performed using CLC Genomics Workbench. Run 2 proved to be a better primer design in every measure and resulted in altered microbial abundance and diversity.

Improved primer effectiveness in Run 2 The primers used in Run 1 and 2 target the same 16S rDNA V3-V4 region (Figure 1 \& Table 1). Consequently, a RDP in silico assay showed that the primer coverage of the microbiome was identical between runs. Both runs were expected to cover approximately $53.86 \%$ of all known bacteria, including $52.61 \%$ of Bacteroidetes, $67.24 \%$ of Firmicutes, $53.76 \%$ of Proteobacteria and $52.66 \%$ of Actinobacteria.

Despite having the same primer coverage, Run 2 primers had improved primer effectiveness and resulted in higher quality

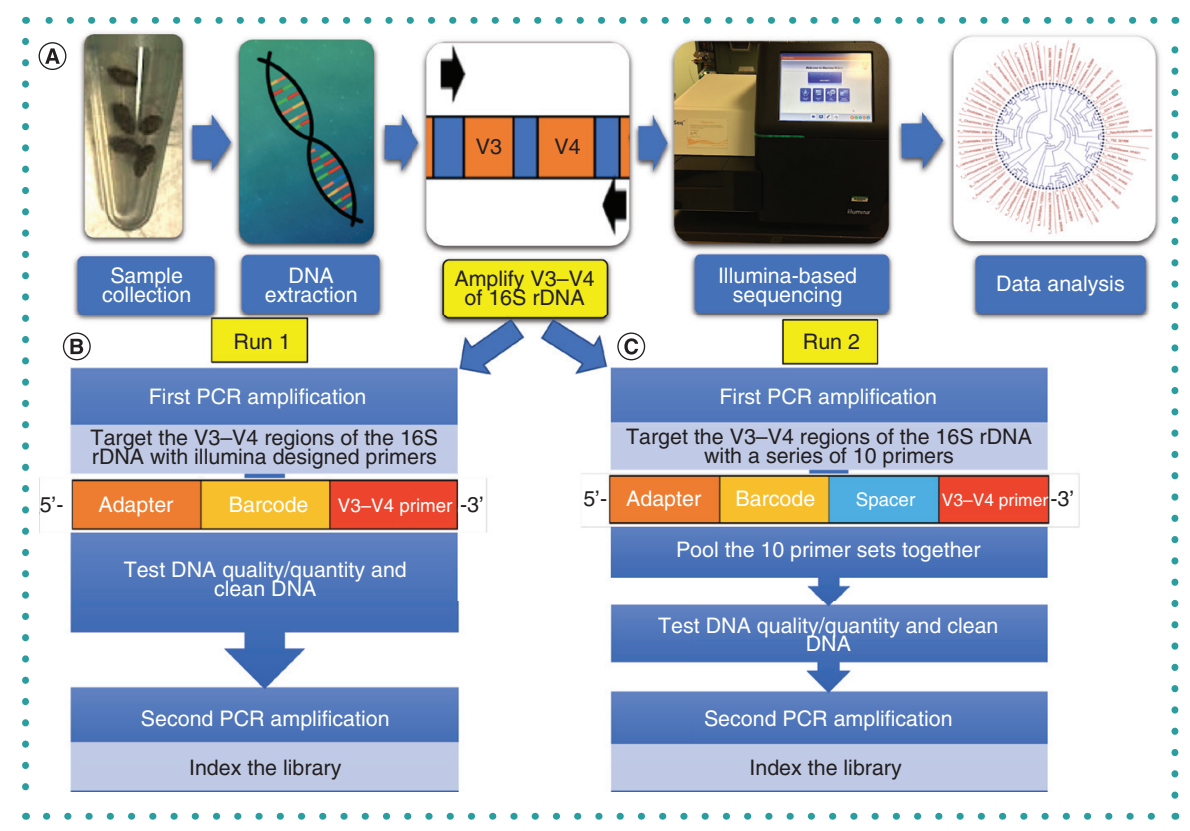

Figure 1. Schematic diagram of 16S rDNA sequencing workflow for both Run 1 and Run 2. (A) The overall workflow for both runs. In brief, bacterial DNA was isolated from fecal pellets using the PowerFecal DNA isolation kit per the manufacturer's protocol. For both runs, the V3-V4 regions of 16S rDNA homologous to bacteria with variable regions unique to microbial species were targeted, and 16S rDNA sequencing was performed using Illumina MiSeq and analyzed for quality. Microbial abundance and diversity were quantified using both QIIME 2.0 and CLC Genomics Workbench Microbial Genomics Module. (B) Targeting V3-V4 regions of 16S rDNA in Run 1. (C) Targeting V3V4 regions of 16S rDNA in Run 2, which amplified the V3-V4 regions through a series of 10 primer sets that include an additional heterogeneity spacer sequence to introduce variability and increase quality of the sequences.

sequencing within the amplicons. PHRED quality scores ( $Q$ score) approximate the confidence level in calling nucleotide bases within the microbial sequences with a goal of Q30 (99.9\% confidence) or greater $[19,20]$. In nearly all sequencing, regardless of chemistry, quality scores drop in later cycles of the run; however, this drop is particularly evident in $2 \times 300$ bp chemistry used with Illumina [21]. This phenomenon was observed in Run 1. The drop in quality occurred at position $60-64$ in both the forward and reverse reads of Run 1, while in Run 2, the drop occurred at position 185 for the forward read and position 160 for reverse read (Figure $2 \&$ Supplementary Figure 1). Moreover, the Q scores in Run 2 were higher in all sequences (a range between 10 and 37 [out of 38] and mode of 37), compared to Run 1 (a range between 8 and 26 and mode of 23).

The overall increase in sequence quality of Run 2 resulted in 98 out of 100 samples passing initial QC (filtering for quality, length and number of sequences, and ability to merge paired-end sequences). In Run 1 , $25 \%$ of samples $(48 / 192)$ failed to meet the requirements for analysis with CLC Genomics Workbench.

To illustrate this difference in how sequence quality affected the number of reads and microbial analysis, a select number of the same samples were analyzed for both Run 1 and 2. Run 2 originally had $45 \%$ more reads from the Illumina run and lost fewer reads during QC; thus, the number of final reads in OTUs was increased by 11-fold in Run 2 versus Run 1 (Table 2). The percentage of retained reads for Run 2 (26.8; 0.511\%) was significantly higher than Run 1 (3.61; 0.41\%; $p<0.001)$. This increase in retained OTU reads and percentage of analyzed samples in Run 2 was expected due to increased variability and confidence in calling nucleotides. Two other studies have utilized the concept of the heterogeneity spacers (one for V3-V4 primers with Illumina MiSeq for clinical samples and the other using Illumina HiSeq for mock microbial communities and environmental samples) along with multiple in-line indexing; both demonstrated an increased quality of sequences and total number of reads $[14,15]$. Mock microbial commu- 


\begin{tabular}{|c|c|c|}
\hline Primers & Direction & Primer sequence \\
\hline \multirow[t]{2}{*}{16.0} & For. & TCGTCGGCAGCGTCAGATGTGTATAAGAGACAGACTCCTACGGGAGGCAGCAG \\
\hline & Rev. & GTCTCGTGGGCTCGGAGATGTGTATAAGAGACAGCCTGGA GGGACTACHVGGGTWTCTAAT \\
\hline \multirow[t]{2}{*}{16.1} & For. & ICGTCGGCAGCGTCAGATGTGTATAAGAGACAGT ACTCCTACGGGAGGCAGCAG \\
\hline & Rev. & GTCTCGTGGGCTCGGAGATGTGTATAAGAGACAGGAGTGG GGACTACHVGGGTWTCTAAT \\
\hline \multirow[t]{2}{*}{16.2} & For. & TCGTCGGCAGCGTCAGATGTGTATAAGAGACAGGTACTCCTACGGGAGGCAGCAG \\
\hline & Rev. & GTCTCGTGGGCTCGGAGATGTGTATAAGAGACAGCACTTCT GGACTACHVGGGTWTCTAAT \\
\hline \multirow[t]{2}{*}{16.3} & For. & TCGTCGGCAGCGTCAGATGTGTATAAGAGACAGCGA ACTCCTACGGGAGGCAGCAG \\
\hline & Rev. & GTCTCGTGGGCTCGGAGATGTGTATAAGAGACAGTTCTCT GGACTACHVGGGTWTCTAAT \\
\hline \multirow[t]{2}{*}{16.4} & For. & TCGTCGGCAGCGTCAGATGTGTATAAGAGACAGATGAA CTCCTACGGGAGGCAGCAG \\
\hline & Rev. & GTCTCGTGGGCTCGGAGATGTGTATAAGAGACAGACTCA GGACTACHVGGGTWTCTAAT \\
\hline \multirow[t]{2}{*}{16.5} & For. & TCGTCGGCAGCGTCAGATGTGTATAAGAGACAGTGCG AACTCCTACGGGAGGCAGCAG \\
\hline & Rev. & GTCTCGTGGGCTCGGAGATGTGTATAAGAGACAGGATA GGACTACHVGGGTWTCTAAT \\
\hline \multirow[t]{2}{*}{16.6} & For. & TCGTCGGCAGCGTCAGATGTGTATAAGAGACAGTGCGT ACTCCTACGGGAGGCAGCAG \\
\hline & Rev. & GTCTCGTGGGCTCGGAGATGTGTATAAGAGACAGCTA GGACTACHVGGGTWTCTAAT \\
\hline \multirow[t]{2}{*}{16.7} & For. & TCGTCGGCAGCGTCAGATGTGTATAAGAGACAGGAGTGG ACTCCTACGGGAGGCAGCAG \\
\hline & Rev. & GTCTCGTGGGCTCGGAGATGTGTATAAGAGACAGTC GGACTACHVGGGTWTCTAAT \\
\hline \multirow[t]{2}{*}{16.8} & For. & TCGTCGGCAGCGTCAGATGTGTATAAGAGACAGCCTGTGG ACTCCTACGGGAGGCAGCAG \\
\hline & Rev. & GTCTCGTGGGCTCGGAGATGTGTATAAGAGACAGA GGACTACHVGGGTWTCTAAT \\
\hline \multirow[t]{2}{*}{16.9} & For. & ICGTCGGCAGCGTCAGATGTGTATAAGAGACAGCCTGGAG ACTCCTACGGGAGGCAGCAG \\
\hline & Rev. & GTCTCGTGGGCTCGGAGATGTGTATAAGAGACAGGGGACTACHVGGGTWTCTAAT \\
\hline
\end{tabular}

Table 2. Sequence yield in Run 1 and Run 2 of the two groups.

\begin{tabular}{|c|c|c|c|c|c|c|}
\hline \multirow[t]{2}{*}{ Samples } & \multicolumn{2}{|c|}{ Number of reads } & \multicolumn{2}{|c|}{ Filtered/chimeric reads } & \multicolumn{2}{|c|}{ Reads in OTUs } \\
\hline & Run 1 & Run 2 & Run 1 & Run 2 & Run 1 & Run 2 \\
\hline Control 1 & 48,034 & 87,936 & 22,264 & 16,920 & 1753 & 22,537 \\
\hline Control 2 & 64,702 & 99,620 & 29,705 & 17,476 & 2646 & 27,824 \\
\hline Control 3 & 61,616 & 106,728 & 29,713 & 20,224 & 1095 & 27,152 \\
\hline Experimental 1 & 66,928 & 85,632 & 30,930 & 15,115 & 2534 & 24,562 \\
\hline Experimental 2 & 71,872 & 101,080 & 33,175 & 18,395 & 2761 & 27,117 \\
\hline Experimental 3 & 102,256 & 94,802 & 46,384 & 17,680 & 4744 & 25,373 \\
\hline Average/run & $69,235 \pm 7373$ & $95,966 \pm 3306$ & $32,029 \pm 7923$ & $17,635 \pm 1683$ & $2589 \pm 504$ & $25,761 \pm 817$ \\
\hline
\end{tabular}

Number of original sequence reads, filtered or chimeric reads and final number of reads in OTUs for select mouse samples in the two groups for the first Illumina and second Illumina run.

OTU: Operational taxonomical unit.

$\checkmark$ nities in 16S rDNA sequencing acts as potential internal controls to test primer effectiveness against a known bacterial composition [22], and in the study with

Vol. $67 \mid$ No. 2 | ๑ 2019 William H Broach
HiSeq, heterogeneity spacers and triple indexing more accurately identified the expected bacterial composition of the mock community [15]. Our study shows that heterogeneity spacers alone can improve sequence quality in the conserved mouse microbiome compared to our controls, Run 1. 

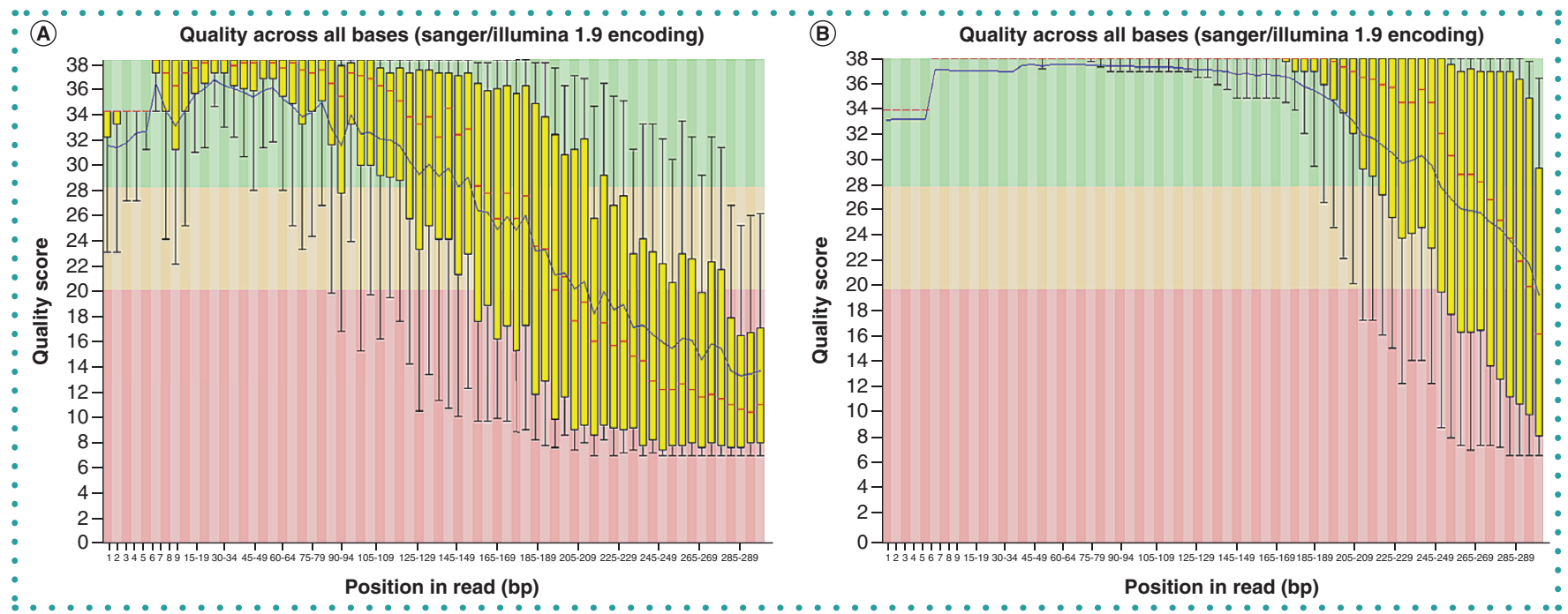

Figure 2. Primer effectiveness. (A) Run 1 of the forward reads with 1 set of primers. (B) Run 2 of the forward reads with the set of 10 primers. (B) Shows less variability in $Q$ scores and higher quality (as seen in the slower drop-off rate at the 185-189 position in read compared to the 60-64 position in read in $(A)$.
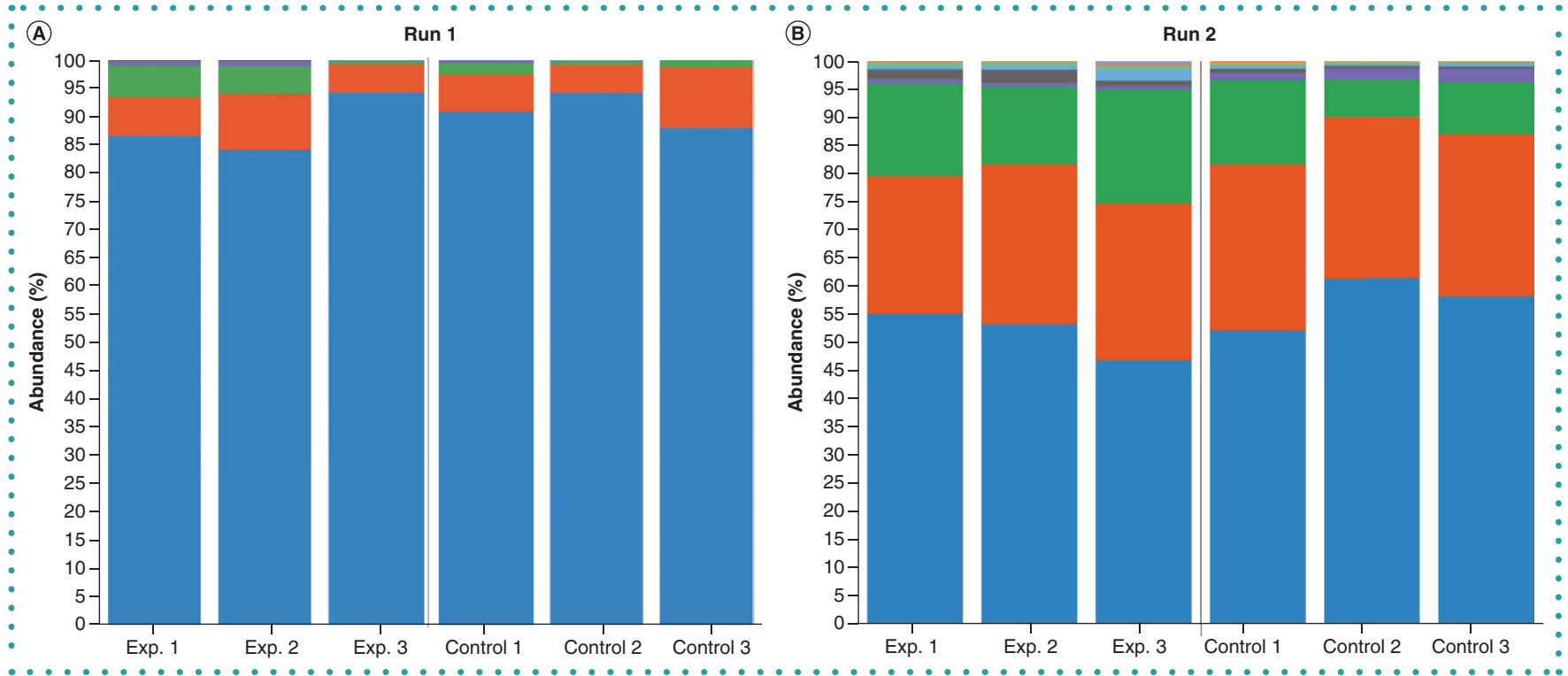

Figure 3. Microbial abundance at the phyla level in both Run 1 and Run 2. (A) Exp and control groups in Run 1. (B) Exp and control groups in Run 2. The most abundant phyla include Bacteroidetes (blue), Firmicutes (orange), Proteobacteria (green), Actinobacteria (purple) and N/A (gray). (B) Increased phyla variation with a higher abundance in the Firmicutes and Proteobacteria phyla. Moreover, (B) shows a clear differentiation in phyla abundance between the experimental and control groups.

Exp: Experimental.

\section{Increased abundance variability}

The difference in sequence quality and number of total OTU reads altered the observed microbial abundance measured in Run 2 compared to Run 1 (Figure 3). In Run 1, Bacteroidetes was the dominant phyla seen in the gut microbiome, consisting of between 84 and $93 \%$ of the total microbial composition. This finding was contrary to what we expected and what is known in the literature, as both Bacteroidetes and Firmicutes typically dominate the mouse and human gut microbiome $[6,23]$. Run 2, however, exhibited a more equalized distribution of the major bacterial phyla. The observed relative abundance of Bacteroidetes was lower, ranging between 47 and $60 \%$, and Firmicutes, Proteobacteria and Actinobacteria had a higher observed relative abundance compared to Run 1. Samples in Run 2 demonstrated increased similarity in the microbial composition at the phyla level by their defined groups. These findings corroborate that increased sequence variability (as seen in Run 2) yields an altered observed microbial abundance [15].

Improved detection of diversity

Run 2 demonstrated an increase in observed microbial diversity - that is, alpha and beta diversity - compared to Run 1. Alpha diversity measures microbial richness (the number of microbial populations) and evenness (proportionality of the microbial populations) of an individual microbial 

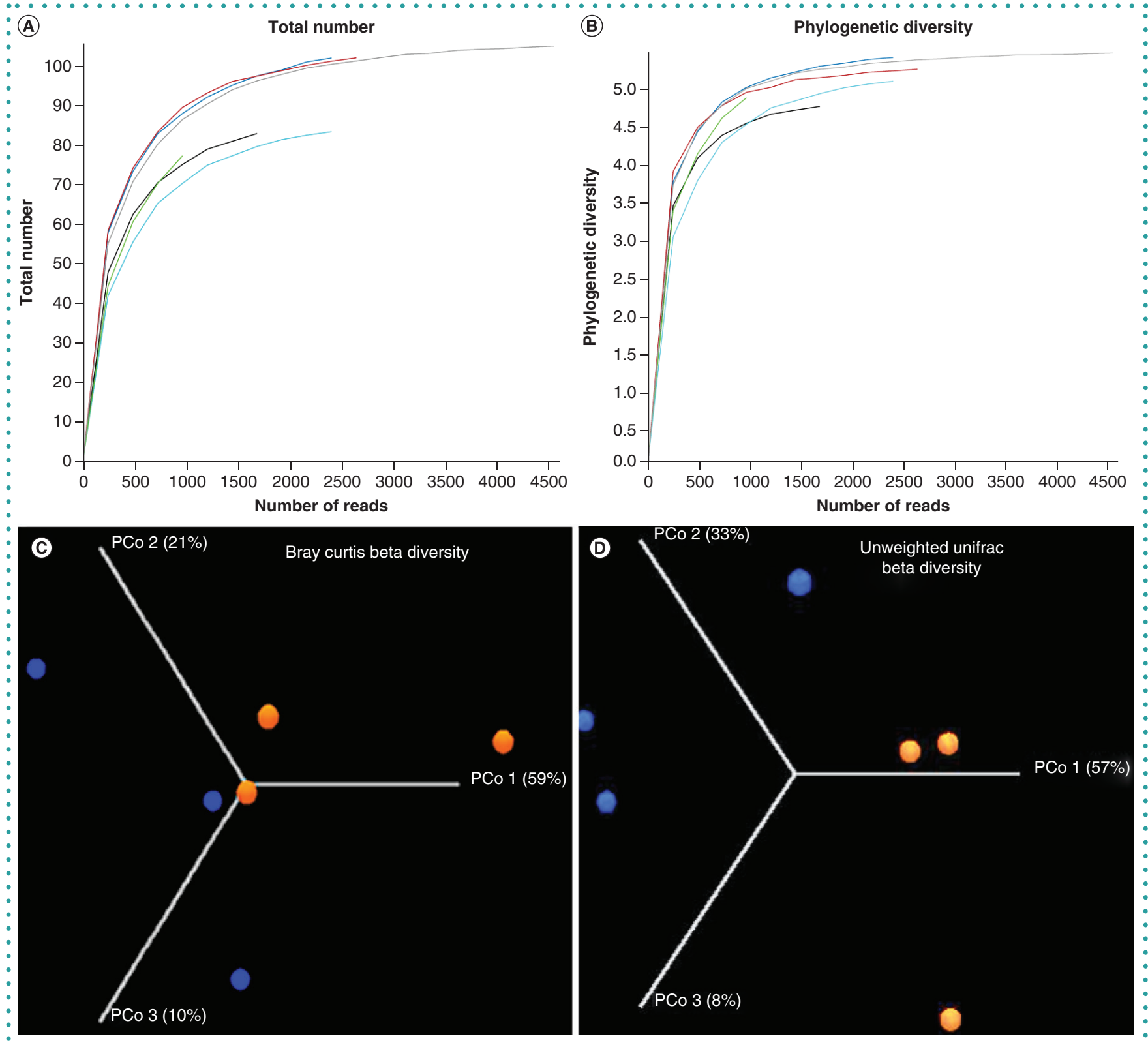

Figure 4. Alpha and beta diversity in the first and second Illumina run for the six selected mouse samples. (A-D) Alpha and beta diversity of Run 1. (A) Total number of operational taxonomical units (OTUs). (B) Phylogenetic diversity. (C \& D) Beta diversity for select mouse samples in the experimental (orange) and control groups (blue; $n=3$ ). (C) Bray-Curtis non-phylogenetic beta diversity (Pseudo-f-statistic $=4.13 ; p=0.10$ ). (D) Unweighted UniFrac phylogenetic beta diversity (Pseudo-f-statistic $=2.26 ; p=0.10$ ). $(E-H)$ Alpha and beta diversity of Run 2. $(E)$ Total number of OTUs. $(F)$ Phylogenetic diversity. (G) Bray-Curtis non-phylogenetic beta diversity (Pseudo-f-statistic $-6.03 ; p=0.10)$. $(H)$ Unweighted UniFrac phylogenetic beta diversity (Pseudo-f-statistic $=13.68 ; p=0.10$ ). Overall, Run 2 demonstrated higher alpha diversity and better differentiation between groups (beta diversity) compared to Run 1.

- community (one sample, such as a single fecal pellet). Four different alpha diversity measurements were quantified, specifically total number of OTUs, phylogenetic diversity, Chao 1 bias-corrected diversity and Shannon entropy. The total number accounts for the total bacterial OTUs for each sample. Run 2 exhibited threefold higher total number of bacterial OTUs (average 340 OTUs; range of 330-350 OTUs) compared to Run 1 (average
100 OTUs, range of 82-104 OTUs) (Figure $4 \mathrm{~A} ; 4 \mathrm{E})$. Phylogenetic diversity, a measurement of the biodiversity of the microbial community integrating the phylogenetic differences between OTUs, was higher in Run 2 (average 7.0) compared to Run 1 (average 5.0) (Figure 4B \& F). Likewise, all samples in Run 2 exhibited higher diversity as quantified by Chao 1 biascorrected diversity (Supplementary Figure
2), a richness measurement based on OTU abundance, and Shannon entropy (Supplementary Figure 2), which accounts for species richness and evenness.

Run 2 had an altered beta diversity compared to Run 1. Beta diversity measures the microbiome differences within and between groups on a distance metric. Four different tests were used to calculate beta diversity, including Jaccard and Bray- 


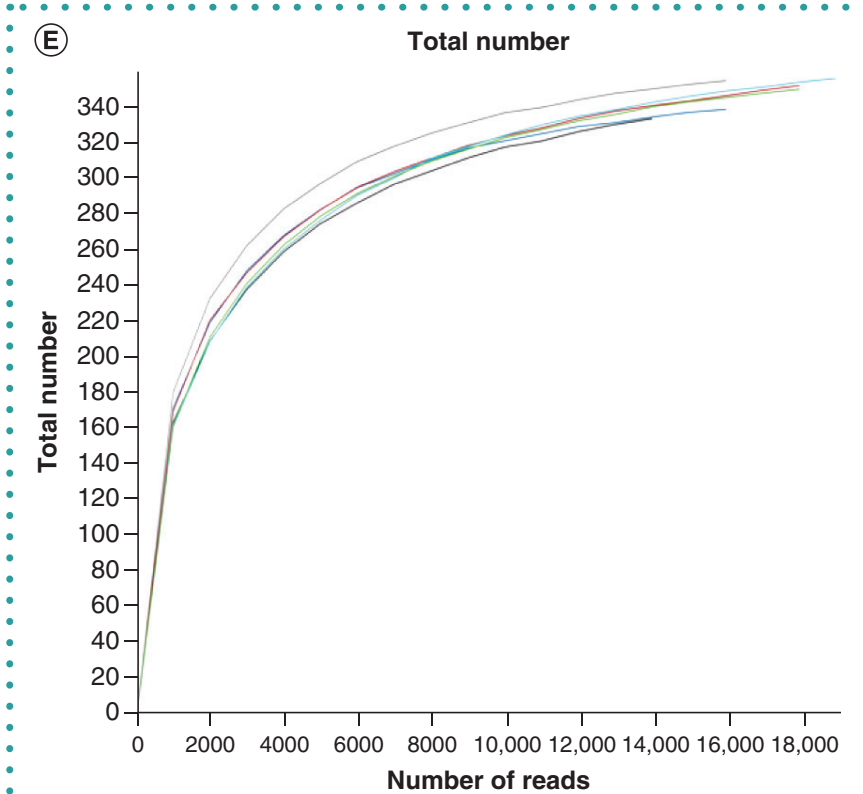

(F) Phylogenetic diversity
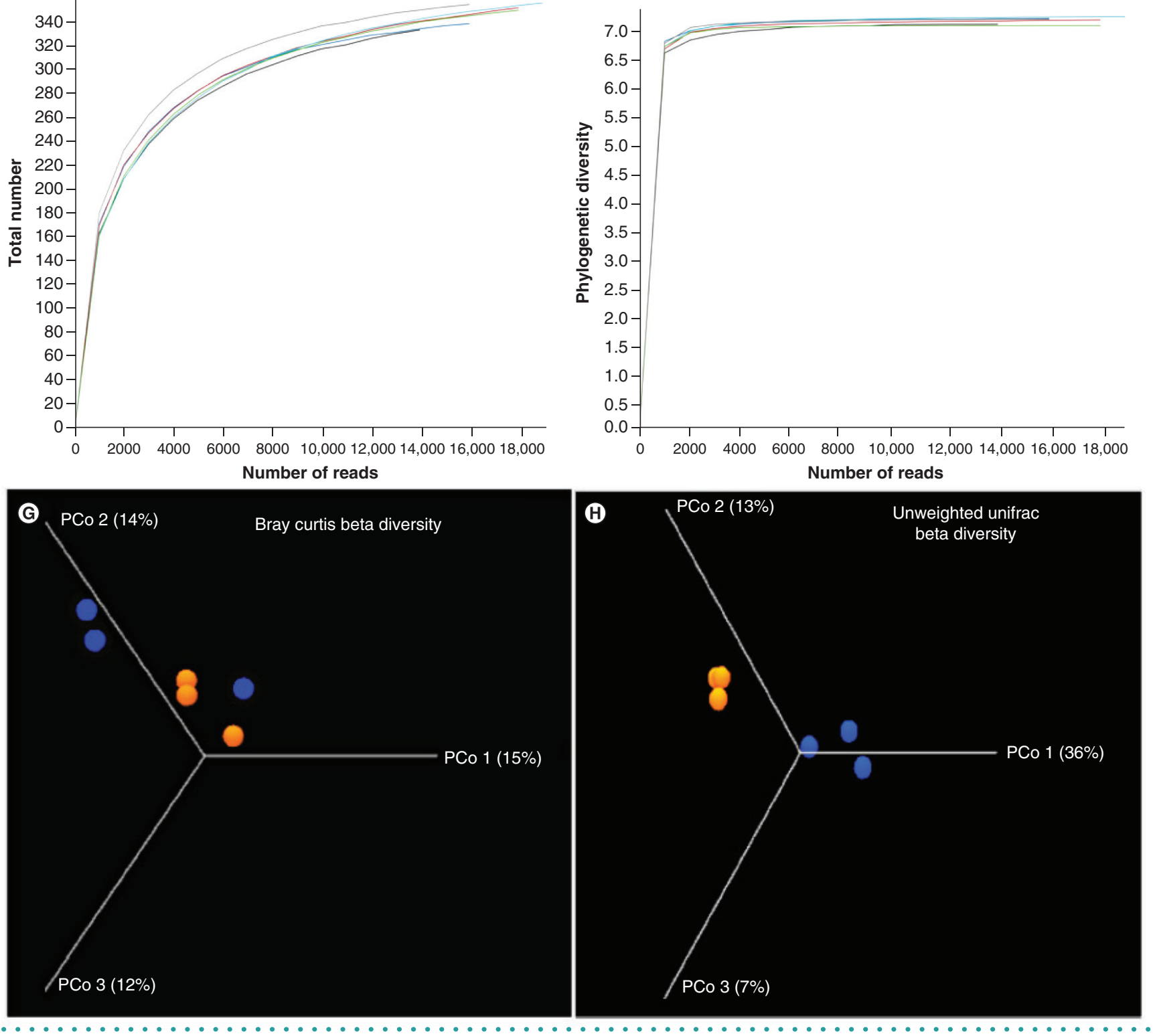

Figure 4. Alpha and beta diversity in the first and second Illumina run for the six selected mouse samples (cont.). (A-D) Alpha and beta diversity of Run 1. (A) Total number of operational taxonomical units (OTUs). (B) Phylogenetic diversity. (C \& D) Beta diversity for select mouse samples in the experimental (orange) and control groups (blue; $n=3$ ). (C) Bray-Curtis non-phylogenetic beta diversity (Pseudo-f-statistic = 4.13; $p=0.10)$. (D) Unweighted UniFrac phylogenetic beta diversity (Pseudo-f-statistic $=2.26 ; p=0.10)$. $(E-H)$ Alpha and beta diversity of Run 2 . (E) Total number of OTUs. $(F)$ Phylogenetic diversity. (G) Bray-Curtis non-phylogenetic beta diversity (Pseudo-f-statistic - 6.03; $p=0.10$ ). (H) Unweighted UniFrac phylogenetic beta diversity (Pseudo-f-statistic $=13.68 ; p=0.10$ ). Overall, Run 2 demonstrated higher alpha diversity and better differentiation between groups (beta diversity) compared to Run 1.

Curtis non-phylogenetic measurements, which measures the taxonomic differences between microbial communities, and weighted and unweighted UniFrac phylogenetic tests, which accounts for the phylogenetic relationship between OTUs in the microbiome (Figure 4C \& D; Figure $4 \mathrm{G} \& \mathrm{H}$; Supplementary Figure 3). Run 2 demonstrated an increase in observed beta diversity in two different ways: 1) increased precision within each experimental group and 2) increased difference between groups. For all beta diversity measurements (except for the weighted UniFrac beta diversity test; Supplemental Figure 3), Run 2 had a larger observed difference between groups (Pseudo-fstatistic, PERMANOVA). Although this difference was drastic between groups, this finding was not statistically signif- icant $(p=0.10)$, most likely due to the small sample number.

Overall, the increase in both alpha and beta diversity of Run 2 corresponds well with the increased data quality seen in previous studies with heterogeneity spacers $[14,15]$. Furthermore, the increased precision within the two groups and distinction between groups demonstrates the power of using heterogeneity spacers 
- for samples with low nucleotide diversity. Additionally, these results demonstrate that an internal or inline index is not required to achieve these benefits.

\section{CONCLUSION}

Overall, our custom-designed set of primers with additional heterogeneity spacers introduces sequence variability into the reads, which is crucial in correctly identifying clusters on the flow cell and improving confidence in nucleotide base calls. Use of our novel primers resulted in improved sequence quality by maximizing the number of reads generated in each sequencing run, as well as an increase in the overall confidence and quality of each nucleotide call. This improvement in sequence quality resulted in an altered observed microbial composition with a more even abundance in the major phyla, increased precision within groups and differentiation between groups as seen in beta diversity. This methodology is a simple and cost-effective way to improve the accuracy and precision in low-diversity samples using $16 \mathrm{~S}$ rDNA sequencing in the V3-V4 region with Illumina MiSeq. Due to its design independent of the binding specificity or adapter regions, heterogeneity spacers can be applied to analyses of the other 16S rDNA hypervariable regions as well as any amplicon sequencing analysis.

\section{FUTURE PERSPECTIVE}

This novel 16S rDNA V3-V4 primer design with the addition of heterogeneity spacers is a simple and cost-effective tool for increasing sequencing variability into reads to ultimately improve accuracy and precision in low diversity samples. Future experiments will include testing these primers with mock microbial communities. Future research is needed to examine the efficacy and effectiveness of this design with other hypervariable regions within the $16 \mathrm{~S}$ rDNA as well as any other amplicon sequencing analyses, including metabolomic studies.

\section{AUTHOR CONTRIBUTIONS}

All authors contributed considerably to the creation of this work. EAJ, DEB, ERM, RKC, EOL and WHB helped in the study design of the project and contributed to troubleshooting for the project. EAJ and JB collected the data and performed the DNA isolation and first round of PCR amplifications. WHB designed the novel primers and performed the rest of the experiments, including the $16 \mathrm{~S}$ rDNA library preparation and amplicon sequencing with Illumina. EAJ and WHB analyzed the data and wrote the draft of the manuscript. All authors contributed to the writing of the manuscript, reviewed, and approved the submitted manuscript.

\section{ACKNOWLEDGMENTS}

We would like to acknowledge Ohio University for the use of their facilities, the assistance and advice of the Genomics Facility at Ohio University and Kaitlin Baker for collecting fecal pellets and helping in the process of DNA isolation from the fecal pellets.

\section{FINANCIAL \& COMPETING INTERESTS DISCLOSURE}

This study was funded by the John $\mathrm{J}$ Kopchick Molecular and Cellular Biology/ Translational Biomedical Sciences Faculty Support Fund and with the assistance of the Office of Research and Grants at Ohio University Heritage College of Osteopathic Medicine and VP of Research and Grants at Ohio University. The authors have no other relevant affiliations or financial involvement with any organization or entity with a financial interest in or financial conflict with the subject matter or materials discussed in the manuscript apart from those disclosed.

No writing assistance was utilized in the production of this manuscript.

\section{ETHICAL CONDUCT OF RESEARCH}

The authors state that this project obtained the appropriate institutional approval for animal experimental investigations. All procedures involving mice were approved by the Ohio University Institutional Animal Care and Use Committee and fully complied with federal, state and local policies.

\section{SUPPLEMENTARY DATA}

To view the supplementary data that accompany this paper please visit the journal website at: www.future-science. com/doi/suppl/10.2144/btn-2019-0025

\section{OPEN ACCESS}

This work is licensed under the AttributionNonCommercial-NoDerivatives 4.0 Unported License. To view a copy of this license, visit http://creativecommons.org/licenses/ by-nc-nd/4.0/

\section{REFERENCES}

1. Bäckhed F, Fraser CM, Ringel $Y$ et al. Defining a healthy human gut microbiome: current concepts, future directions, and clinical applications. Cell Host $\&$ Microbe. 12(5), 611-622 (2012).

2. Gilbert JA, Blaser MJ, Caporaso JG, Jansson JK, Lynch SV, Knight R. Current understanding of the human

3. Brooks JP, Edwards DJ, Harwich MD et al. The truth about metagenomics: quantifying and counteracting (2015).

4. Kozich JJ, Westcott SL, Baxter NT, Highlander SK, Schloss PD. Development of a dual-index sequencing strategy and curation pipeline for analyzing amplicon sequence data on the MiSeq Illumina sequencing platform. Appl. Environ. Microbiol. 79(17), 5112-5120 (2013).

5. D'Amore $\mathrm{R}$, ljaz UZ, Schirmer $\mathrm{M}$ et al. A comprehensive benchmarking study of protocols and sequencing platforms for 16S rRNA community profiling. BMC Genomics 17(1), 55 (2016)

6. Thijs S, Op De Beeck M, Beckers B et al. Comparative evaluation of

7. Kim M, Morrison M, Yu Z. Evaluation of different partial $16 \mathrm{~S}$ rRNA gene sequence regions for phylogenetic analysis of microbiomes. J. Microbiol. Methods 84(1), 81-87 (2011)

8. Klindworth A, Pruesse E, Schweer T et al. Evaluation of general 16S ribosomal RNA gene PCR primers for classical and next-generation sequencing-based diversity studies. Nucleic Acids Res. 41(1), e1 (2013).

9. Lu Y-Z, Ding Z-W, Ding J, Fu L, Zeng RJ. Design and evaluation of universal 16S rRNA gene primers for high-throughput sequencing to simultaneously detect 385-394 (2015).

10. Shin J, Lee S, Go M-J et al. Analysis of the mouse gut microbiome using full-length 16S rRNA amplicon sequencing. Sci. Rep. 6, 29681 (2016)

11. Castelino M, Eyre S, Moat J et al. Optimisation of methods for bacterial skin microbiome investigation: primer selection and comparison of the 454 versus MiSeq platform. BMC Microbiol. 17(1), 23 (2017).

12. Rintala A, Pietilä S, Munukka E et al. Gut microbiota analysis results are highly dependent on the 16S rRNA gene target region, whereas the impact of DNA extrac 28(1), 19-30 (2017)

13. Schirmer M, ljaz UZ, D'Amore R, Hall N, Sloan WT, Quince $C$. Insight into biases and sequencing errors for amplicon sequencing with the Illumina MiSeq platform Nucleic Acids Res. 43(6), e37 (6)

14. Fadrosh DW, Ma B, Gajer P et al. An improved dual-indexing approach for multiplexed 16S rRNA gene sequencing on the Illumina MiSeq platform. Microbiome 2(1), 6 (2014).

15. de Muinck EJ, Trosvik P, Gilfillan GD, Hov JR, Sundaram AYM. A novel ultra high-throughput 16S rRNA gene amplicon sequencing library preparation method for the Illumina HiSeq platform. Microbiome 5(1), 68 (2017).

16. Fuks G, Elgart M, Amir A et al. Combining 16S rRNA gene variable regions enables high-resolution microbal community profiling. Microbiome 6(1), 17 (2018).

17. Caporaso JG, Kuczynski J, Stombaugh J et al. QIIME allows analysis of high-throughput community se-
quencing data. Nat. Methods 7(5), 335-336 (2010).

18. Quast C, Pruesse E, Yilmaz $P$ et al. The SILVA ribosomal RNA gene database project: improved data processing and web-based tools. Nucleic Acids Res. 41(D1), D590-D596 (2013).

19. Ewing B, Hillier L, Wendl MC, Green P. Base-calling of assessment. Genome Res. 8(3), 175-185 (1998).

20. Ewing B, Green P. Base-calling of automated sequencer traces using Phred. II. Error Probabilities. Genome Res. 8(3), 186-194 (1998)

21. Sinclair L, Osman OA, Bertilsson S, Eiler A. Microbial community composition and diversity via $16 \mathrm{~S}$ rRNA gene amplicons: evaluating the Illumina platform. PLOS One 10(2), e0116955 (2015).

22. Cao $Y$, Fanning S, Proos S, Jordan $K$, Srikumar S. A review on the applications of next generation sequencing studies. Front. Microbiol. 8, 1829 (2017).

23. Nguyen TLA, Vieira-Silva S, Liston A, Raes J. How informative is the mouse for human gut microbiot research? Dis. Model Mech. 8(1), 1-16 (2015). 